\title{
Community Pharmacy Centered Rural Mobile Diabetic Retinopathy Screening Service
}

\author{
Francisco J. Jiménez-Ramírez ${ }^{1 *}$, Jose Josue Hernandez ${ }^{1}$, Raul Perez ${ }^{2}$, Wilmarie Lorenzo-Gonzalez ${ }^{1}$, Yiselis \\ Perez-Caban $^{1}$, Karen N. Soto-Toledo ${ }^{1}$, Tania J. Saavedra-Diaz ${ }^{1}$ and Suzette M. Vélez-Rivera ${ }^{1}$ \\ ${ }^{1}$ Department of Pharmacy Practice, University of Puerto Rico School of Pharmacy, San Juan, Puerto Rico, USA \\ ${ }^{2}$ Department of Ophthalmology, University of Puerto Rico School of Medicine, San Juan, Puerto Rico, USA
}

\begin{abstract}
Received: March 06, 2015; Accepted: April 29, 2015; Published: May 11, 2015
*Corresponding author: Francisco J. Jiménez-Ramírez, Department of Pharmacy Practice, University of Puerto Rico School of Pharmacy, San Juan, Puerto Rico, USA, Tel: +787-758-2525 x 5115; E-mail: Francisco.Jimenez3@upr.edu
\end{abstract}

\begin{abstract}
Diabetic Retinopathy (DR) is considered as one of the leading global causes of blindness. Long-term poor glucose control leads to vascular incompetency, tissue swelling, new vessels formation, and if untreated, to irreversible severe visual loss. The purpose of this study was to demonstrate that interventions at the pharmacy level in remote, underserved, and isolated communities contribute to the early diagnosis and treatment of DR and its related complications. Educational programs and telemedicine retinal screening may expand the pharmacist role to provide health education to encourage self-care and complications screening
\end{abstract}

A descriptive study was performed at six community pharmacies in Puerto Rico. An educational session preceded an interview and a digital image of the retina taken by a trained pharmacist using the transportable i-Optic Easy Scan Camera that uses Scanning Laser Ophthalmoscope technology. Images were sent electronically to a retinologist for assessment and appropriate recommendations. Patients took results to their primary physicians and preferred eye care specialist.

One hundred and sixty-three (163) individuals participated in the educational session phase. Out of these, $128(79 \%)$ completed the study by proceeding to the interview and digital imaging of the retina phase. Participants mean age was 58.2 years, and $58 \%$ were females. Forty (40) participants were classified as having suspected diabetic retinopathy (DR), resulting in an overall DR prevalence of $31 \%$. Background DR (BDR) was identified in $38(95 \%)$ and proliferative DR in $2(5 \%)$ of all the DR classified cases. The study was able to demonstrate that a distant or remote mobile service in community pharmacies is a viable way to provide education and annual eye exams to screen people with diabetes to early identify and treat DR.

Keywords: Diabetic retinopathy; Diabetes mellitus; Community pharmacy

\section{Introduction}

Diabetic Retinopathy (DR) is considered one of the leading global causes of blindness [1]. It is a common complication of Diabetes Mellitus (DM) caused by changes in retinal blood vessels as the result of long-term poor glucose control. In some people with DR, blood vessels may swell and leak fluid, while in others an abnormal growth of new blood vessels on the surface of the retina, known as collateral microcirculation, may occur [2]. DR progresses in several predictable steps or stages. The initial stage of the condition, known as non-proliferative DR may develop and progress without symptoms. Proliferative DR is the last and more serious stage, leading to blindness and disability if not detected and treated in time. Early detection and management of proliferative DR and associated complications such as Diabetic Macular Edema (DME) and retinal detachment is essential to prevent blindness $[3,4]$.

The prevalence of DM in the United States has increased dramatically since 2000, resulting in an increased risk of DR and its complications. In 2010, the Center for Disease Control and Prevention conducted a study that demonstrated that the prevalence of DR affects almost one-third of adults over 40 years old with DM, and more than one-third of African-Americans and Mexican-Americans [5]. In 2010, 1.9 million new cases of DR were diagnosed in people over 20 years of age and older. According to the 2011 National Diabetes Fact Sheet, between 2005-2008, 4.2 million people over 40 years of age with DM were diagnosed with DR, with nearly 700,000 suffering from advanced DR that could lead to severe vision loss and disability [6]. Furthermore, Hispanics/Latinos most commonly present DM related complications and worse outcomes when compared with other ethnic groups [5]. When the burden of DM is compared among subgroups of Hispanics/Latinos born in the US, foreignborn Puerto Ricans exhibit a higher prevalence of hypertension (32\%) and DM (15\%) after adjusting for age, BMI, smoking, socioeconomic status and acculturation [7]. It is estimated that 13 out of every 100 Puerto Ricans older than 20 years of age have DM. Prevalence of DM has increased in Puerto Rico from $9.8 \%$ in 2001 to $12.9 \%$ in 2010 [5]. Perez et al. [7] analyzed data from an epidemiologic study in the metropolitan area of San Juan demonstrating that less than half of people with DM reported an annual dilated eye examination (49.2\%). Without proper and timely screening evaluations and education, the risk of developing DR in patients with DM is high. 
Jimenez et al. [8] found that screening in community pharmacies in Puerto Rico resulted in early diagnosis and treatment of DR and other disabling eye disorders such as glaucoma. Retinal findings associated with DR encouraged and eased its early referral and treatment. In this study, educational sessions at community pharmacies in Barranquitas and Lares lead to early screening of 72 patients. DR was diagnosed in 16 patients $(22.2 \%)$. Of these patients with newly diagnosed DR, $12.5 \%$ ( 2 of 16 patients) were graded as having severe DR in both eyes, $31.2 \%$ ( 5 of 16 patients) had DME, 37.5\% (6 of 16 patients) had background DR and one patient presented with proliferative DR.

Early screening and detection of DR in the local population with DM have many benefits. Although the risk of DR among patients with type 2 DM has increased by factors such as poor glucose control, elevated lipids, and high blood pressure, these factors do not predict which patients will develop DR, even when monitored regularly [9]. Progression of DR can be delayed or arrested if treated during the early stages of the disease, but the condition is often fairly advanced in many patients by the time a diagnosis of type 2 DM is made [9]. Early detection allows prompt treatment, and prevents progression to severe visual loss and permanent disability. Community screening will promote intensive therapy and prevents DR progression. Early identification of patients with DR will result in timely referral for early treatment, decreasing the likelihood of progression to severe disabling visual loss $[4,10]$. In addition, coronary artery disease and diabetic nephropathy will also be promptly detected and treated, lowering the risk for early death, serial dialysis and renal transplantation $[4,10]$.

Pharmacies represent an optimal setting to integrate services that benefit patients with chronic conditions such as DM. Pharmacists, in collaboration with ophthalmologists and other health care professionals, can aid in the early diagnosis and treatment of DR. The pharmacist is the health care professional who is most accessible to the underserved community with DM, for both, day to day contingencies and emergent situations. Pharmacists can play an important role in educational outreach efforts that focus on the prevention and early detection of DR and other conditions. Pharmacists can develop and coordinate services in their communities to educate patients about healthcare problems such as DR, the benefits of glucose control in the prevention of diabetes-related complications, and the importance of an early diagnosis of these complications. Pharmacists, as an integral part of the healthcare team, can easily provide access to the required examinations in this group of patients, and contribute to improve the health and quality of life of the population with DM in Puerto Rico [8].

The purpose of this study was to demonstrate that pharmacist' interventions in community pharmacies could contribute to the identification of people with DM who are at high risk of presenting undiagnosed DR, promoting an early diagnosis and early treatment. Investigators expect pharmacists to be seen in the community as healthcare providers that may improve the quality of life of patients with DM and are committed in the implementation of strategies aimed to prevent the development of DM related complications and progression, such as DR. Therefore, creating patient awareness regarding the importance of having an annual dilated eye examination to early diagnose and treat DR is one of the main goals of this study.

The objectives of this study were to: 1) Demonstrate that pharmacists are capable, through focused educational interventions, to provide patients with DM with up to date medical knowledge essential for their self-care and reinforcing the relevance of DR screening. 2) Demonstrate that community pharmacies are ideal settings to provide access to annual eye exam for early detection of DR and referral to treatment in underserved, impoverished, rural and minority populations. 3) Refer newly diagnosed patients with DR to their preferred eye care specialist for appropriate treatment. 4) Demonstrate the impact that pharmacists as health care providers may have in the detection, prevention and education of chronic health conditions and related complications, in collaboration with other health care providers as eye care specialists.

\section{Methods}

Six different community pharmacies in Puerto Rico were used to recruit the study participants, provide the educational services and perform the eye examination. Individuals 21 years of age or older with an established diagnosis of type 2 DM or with type 1 DM of at least five years duration, were eligible to participate in the study. Patients who met the inclusion criteria were identified by the pharmacy staff of each of the six community pharmacies. Pharmacists offered potential participants the opportunity to participate in the study and gave them an information sheet with details related to the study. Participants currently under the care of an ophthalmologist for previously diagnosed DR, or having a life expectancy shorter than 6 months were excluded from the study. All participants signed an informed consent provided by one of the researchers. Community pharmacies were responsible of both, coordination and promotion of the educational and assessment sessions, and educating the community with DM. Pharmacists were responsible for recruiting potential participants and providing them with the information sheet describing the study. They were also responsible for notifying the physicians who would receive the ophthalmologist's assessment (diagnosis) and recommendations.

The research group, comprised of the ophthalmologist, a licensed pharmacist and three Doctor of Pharmacy (Pharm.D.) candidates, developed the DR educational session following the American Diabetes Association (ADA) recommendations [11]. Prior to the screening evaluation, all participants received the educational intervention offered by a pharmacist from the research group. Two interactive educational lectures with time for peers (patients) experiences sharing and questions and answers were scheduled during the day, at 8:00 am for the morning session and at 1:00 pm for the afternoon session. The educational lectures were developed with the purpose of enhancing awareness about the importance of glucose control in people with DM in order to prevent diabetes-related complications and the importance of an annual eye examination for the early diagnosis and treatment of 
DR. The educational lectures content included information about risk factors, prevention, treatment, importance of DM control and appropriate management of other comorbidities such as hypertension and dyslipidemia.

After signing an informed consent, patients who met the inclusion criteria were interviewed by one of the study researchers to collect their health profile, including their DM treatment and history. The following data was recorded: sex, age, weight, height, waist and hip circumference, type of DM, duration of DM, and overall pharmacologic therapy. Also, clinical examination including levels of fasting plasma glucose, and glycosylated hemoglobin $\mathrm{A}_{1 \mathrm{C}}\left(\mathrm{HbA}_{1 \mathrm{c}}\right)$ were collected.

A DR screening service was available after the educational session for the participants who qualified and signed the informed consent. The main purpose of the screening session was to provide access to the annual eye exam to participants and to identify participants with undiagnosed DR. A private office inside the pharmacy was used for the remote retinal image capture using the i-Optic Easy Scan Camera that uses a Scanning Laser Ophthalmoscope technology. The i-Optic Easy Scan company vendor trained a pharmacist to manage the equipment for taking the images. Training included setting up, accommodating the patient, centralizing the images, adjusting and focusing for image quality, recording and data protection. The pharmacist captured one non-dilated retinal images of each eye of each participant at the day of the educational session and assigned an unidentifiable number to each image. Easy Scan is a retinal imaging system that does not require dilation because it uses green and infrared laser technology providing optimal patient comfort. It captures images through small pupils with no dilation required. The system captures 3 images with one shot using green light $(532 \mathrm{~nm})$, near infrared (785 nm) and combined (pseudo color) to enhance diagnosis. The ophthalmologist, who was blinded of the study participants, received the forwarded images remotely captured for grading and assessment. Once an assessment was made, the ophthalmologist sent the assessment to the pharmacy. The pharmacist handed in the ophthalmologist's recommendations to the participants in one week or before after the images were captured. A copy of the ophthalmologist recommendations was given to all patients to take it to their primary physician and preferred eye care specialist. The Easy Scan software allows for patient-record navigation, storage, retrieval (identification number, referring doctor, findings classification, among others) as well as an enhancement tool to assist the ophthalmologist in looking at specific areas of the retina. All data was digitalized and stored in a laptop computer that belonged to the researchers. Access to patient's information was restricted to the research group and a username and password was required to guarantee confidentiality.

Descriptive statistics such as frequency count, percentages, averages and standard deviations were used to analyze demographic and health history data. Baseline characteristics of the study participants with and without DR were compared using chi-square and t-test. A logistic regression model was applied to assess associations between the presence of DR and other parameters. The Hosmer-Lemeshow test for logistic regression was used to verify how well the model fitted the data and Odds Ratios (ORs) and 95\% Confidence Intervals (CIs) were calculated.

\section{Results}

A total of 163 individuals participated in the educational sessions provided at any of the six community pharmacies. Out of these 163 participants, 12 met the following exclusion criteria; $6(50 \%)$ patients had previous laser therapy, $2(16.7 \%)$ were younger than 21 years of age, and $4(33.3 \%)$ did not have a confirmed diagnosis of DM. Out of the remaining 151 participants, a total of 8 refused to sign an informed consent form and 15 did not complete the patient interview. The total final sample size was of 128 individuals (Figure 1). The mean age of the participants was 58.2 years (SD 12.5), and 74 (58\%) were females. A total of 40 participants were classified as having suspected DR, resulting in an overall prevalence of 31\% (Table 1). Background DR (BDR) was suspected in 38 (95\%) and proliferative DR (PDR) in 2 (5\%) of the participants classified as having DR. A total of 160 eye disorders were newly diagnosed in 101 (79\%) of the 128 participants (Figure 2). A diagnosis of glaucoma was suggested by the ophthalmologist through the identification of changes in the optic nerve in 52 (40.6\%) participants without a prior history of the disease, out of these 52; twelve were also diagnosed with DR. Intraocular pressure was not measured at this time.

Among the group of 40 subjects with DR the mean age was 56.8 years (SD 11.7), 19 (48\%) were female and the mean duration of the DM was 11.9 years (SD 8.6). In this group, the mean fasting plasma glucose (FPG) was $150 \mathrm{mg} / \mathrm{dL}$ (SD 46.3), the average $\mathrm{HbA}_{1 \mathrm{C}}$ was $7.8 \%$ (SD 2.0) and the mean systolic blood

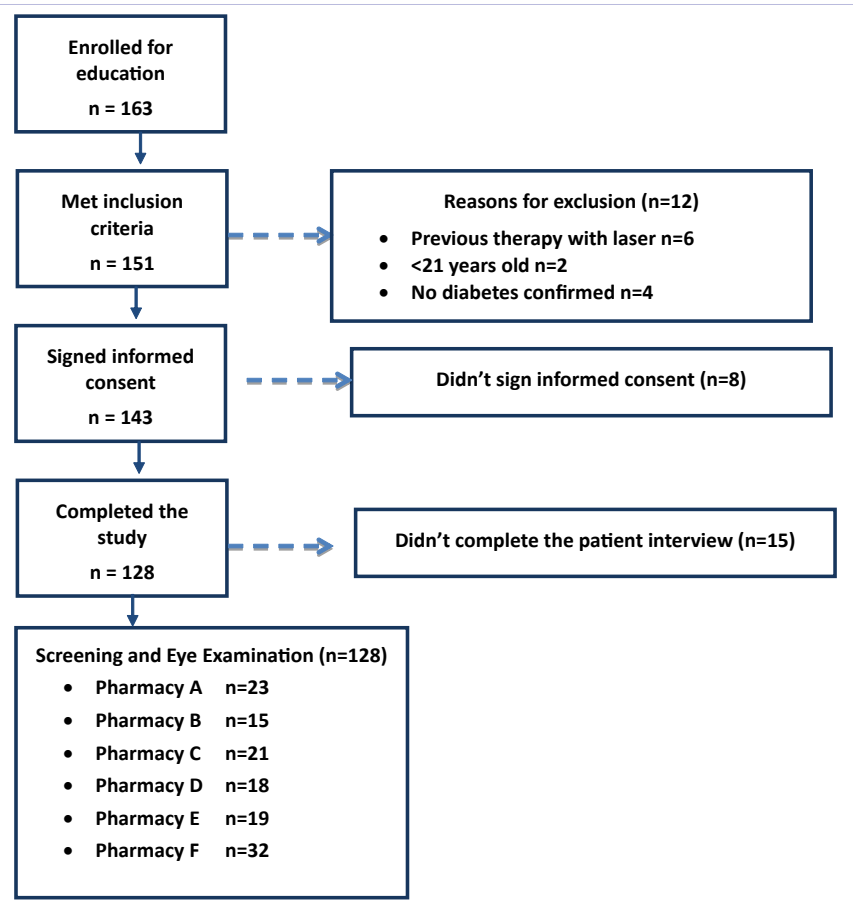

Figure 1: Patient disposition. 
Table 1: Descriptive Statistics for the Study Sample $(n=128)^{*} n=48$ for $\mathrm{HbA}_{1 \mathrm{c}}$

\begin{tabular}{|c|c|}
\hline Variable & Mean \pm SD \\
\hline Age (years) & $58.2 \pm 12.5$ \\
\hline Weight (lb) & $173.4 \pm 38.3$ \\
\hline Height (in) & $64.0 \pm 4.2$ \\
\hline Body Mass Index & $29.8 \pm 6.0$ \\
\hline Waist Circumference (in) & $40.4 \pm 5.4$ \\
\hline Hip Circumference (in) & $42.7 \pm 4.8$ \\
\hline Duration of diabetes (years) & $9.5 \pm 7.4$ \\
\hline Fasting Plasma Glucose (mg/dL) & $152.1 \pm 57.4$ \\
\hline $\operatorname{HbA1c}(\%)^{*}$ & $7.8 \pm 2.2$ \\
\hline Systolic Blood Pressure (mmHg) & $137.2 \pm 19.4$ \\
\hline Diastolic Blood Pressure (mmHg) & $77.6 \pm 10.6$ \\
\hline \multirow[t]{2}{*}{ Heart rate (bpm) } & $77.8 \pm 12.0$ \\
\hline & n (\%) \\
\hline \begin{tabular}{|c|} 
Gender \\
Male \\
Female
\end{tabular} & $\begin{array}{l}54(42) \\
74(58)\end{array}$ \\
\hline $\begin{array}{l}\text { Pharmacy } \\
\text { A } \\
\text { B } \\
\text { C } \\
\text { D } \\
\text { E } \\
\text { F }\end{array}$ & $\begin{array}{l}23(18) \\
15(12) \\
21(16) \\
18(14) \\
19(15) \\
32(25)\end{array}$ \\
\hline $\begin{array}{l}\text { Diabetic Retinopathy } \\
\text { Yes } \\
\text { No }\end{array}$ & $\begin{array}{l}40(31) \\
88(69)\end{array}$ \\
\hline $\begin{array}{l}\text { Diabetic Drugs } \\
\text { Biguanides } \\
\text { Insulins } \\
\text { Sulfonylureas } \\
\text { DPP-4 inhibitors } \\
\text { TZD } \\
\end{array}$ & $\begin{array}{c}84(66) \\
55(43) \\
47(37) \\
15(12) \\
6(5)\end{array}$ \\
\hline $\begin{array}{l}\text { Hypertension Drugs } \\
\text { ACE-I/ARB } \\
\beta \text {-blockers } \\
\text { Thiazides } \\
\text { CCB } \\
\text { Loop diuretics } \\
\text { Central } \alpha_{2-} \text { agonists } \\
\alpha_{1 \cdot} \text { adrenergic blockers }\end{array}$ & $\begin{array}{l}92(72) \\
21(16) \\
16(13) \\
14(11) \\
10(8) \\
4(3) \\
3(2)\end{array}$ \\
\hline $\begin{array}{l}\text { Hyperlipidemia Drugs } \\
\text { Statins } \\
\text { Fibrates } \\
\text { Combination } \\
\end{array}$ & $\begin{array}{c}62(48) \\
6(5) \\
1(<1)\end{array}$ \\
\hline \begin{tabular}{|l} 
Antiplatelet Drugs \\
Aspirin \\
Combination \\
Clopidogrel
\end{tabular} & $\begin{array}{c}56(44) \\
6(5) \\
1(<1)\end{array}$ \\
\hline
\end{tabular}

pressure was $143 \mathrm{mmHg}$ (SD 26). A non-adjusted statistical analysis comparing the group of participants with and without DR revealed that duration of diabetes $(P=0.02)$ and systolic blood pressure $(P=0.04)$ were significantly higher in those with $D R$ compared to those without DR. In addition, this analysis revealed that participants with DR have 2.37 times higher odds of having a history of using insulin for the treatment of their DM, (OR,2.37; 95\% CI, 1.10-5.08), 2.93 times higher odds of using Angiotensin Converting Enzyme Inhibitors (ACE-I) or Angiotensin Receptor Blockers (ARB) pharmacologic therapies (OR, 2.93; 95\% CI 1.11-7.76), 6.01 times higher odds of using a loop diuretics (OR, 6.01; 95\% CI, 1.47-24.64), and 2.54 times higher odds of using aspirin (OR, 2.54; 95\% CI, 1.05-4.83) compared with the odds for participants without DR (Table 2).

The age stratified prevalence of DR was $0 \%(0$ of 4 participants) in the age group 21-29 years old, $67 \%$ (4 of 6 participants) in the age group 30-39 years old, 29\% (5 of 17 participants) in the age group 40-49 years old, 29\% (10 of 35 participants) in the age group 50-59 years old, 36\% (17 of 47 participants) in the age group 60-69 years old, and $21 \%$ (4 of 19 participants) in the age group $\geq 70$ years old. Also, the prevalence of DR was 19\% (7 of 37) in participants with a history of DM of $<5$ years, $32 \%$ (11 of 34) in participants with a history of DM of 5-9 years of duration, $28 \%$ (7 of 25 ) for those with a diagnosis of DM of 10-14 years, $40 \%$ (6 of 15) for those with a diagnosis of DM of $15-19$ years, $50 \%$ ( 5 of 10 ) for those with a diagnosis of DM of 20-24 years, and $57 \%$ (4 of 7) in participants with a diagnosis of $\mathrm{DM} \geq 25$ years (Figure 3 ).

As an exploratory tool for future research, an adjusted logistic regression model was carried out with DR as the dependent variable, and age group, gender, body mass index, duration of DM, systolic blood pressure, ACE-I or ARB use, loop diuretic use, and aspirin use as the independent variables (Table 3). The Hosmer and Lemeshow Goodness of Fit statistics demonstrated that the data fitted the specified model ( $P=0.7435)$. The model revealed that the presence of DR was less likely among females than males $(\mathrm{OR}=0.39 ; 95 \% \mathrm{CI} 0.16-0.99)$. It also demonstrated that longer periods of time since diagnosis of DM $(\mathrm{OR}=1.08 ; 95 \%$ CI 1.02-0.1.15), higher systolic blood pressure (OR=1.04; 95\%CI 1.02-1.07) and the use of loop diuretics (OR=6.42; 95\% CI 1.3530.60 ) were all significantly associated with the presence of DR.

\section{Discussion}

One of the main objectives of this study was to demonstrate that pharmacists are capable, through focused educational interventions, to provide patients with DM with up to date medical knowledge essential for their self-care and reinforcing the relevance of DR screening. This objective was clearly achieved as a total of $79 \%$ of the patients with DM who participated in the educational interventions decided to participate in the DR screening phase. The fact that more than three-fourth of the educational intervention participants had an eye screening performed at their community pharmacy serve as evidence that community pharmacies are an ideal setting to provide access to annual eye exam for early detection of DR in underserved, impoverished, rural and minority populations. In addition, the 


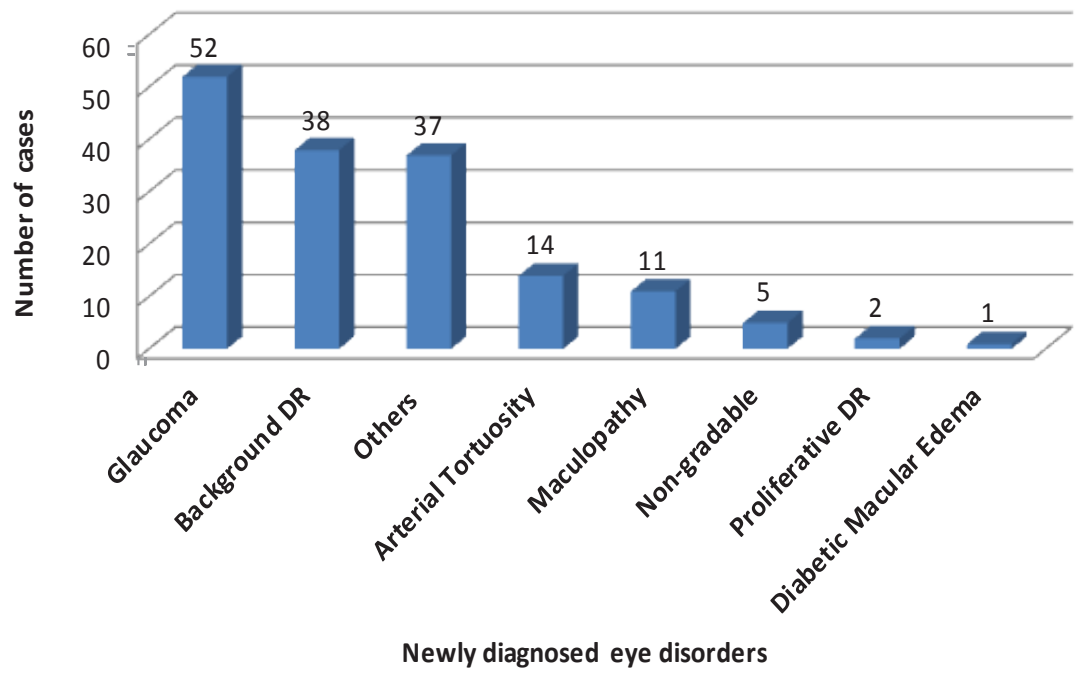

Figure 2: Newly diagnosed eye disorders $(\mathrm{n}=160)$.

Table 2: Differences between the Groups of Subjects with Diabetic Retinopathy Compared with the Group of Subjects without Diabetic Retinopathy $(\mathrm{n}=128)$.

\begin{tabular}{|c|c|c|c|}
\hline & $\begin{array}{l}\text { No Diabetic Retinopathy } \\
\qquad \mathrm{n}=88\end{array}$ & $\begin{array}{c}\text { Diabetic } \\
\text { Retinopathy } \\
\mathrm{n}=40\end{array}$ & \\
\hline Variable & Mean \pm SD & Mean \pm SD & P-value \\
\hline Age (years) & $58.8 \pm 12.8$ & $56.8 \pm 11.7$ & 0.41 \\
\hline Weight (lbs.) & $170.1 \pm 37.9$ & $180.8 \pm 38.8$ & 0.14 \\
\hline Height (in) & $63.7 \pm 4.2$ & $64.7 \pm 4.0$ & 0.21 \\
\hline Body Mass Index & $29.5 \pm 6.0$ & $30.4 \pm 6.0$ & 0.44 \\
\hline Waist Circumference (in) & $40.0 \pm 5.3$ & $41.4 \pm 5.4$ & 0.16 \\
\hline Hip Circumference (in) & $42.5 \pm 4.9$ & $43.2 \pm 4.6$ & 0.47 \\
\hline Duration of diabetes (years) & $8.4 \pm 6.5$ & $11.9 \pm 8.6$ & 0.02 \\
\hline Fasting plasma glucose $(\mathrm{mg} / \mathrm{dL})$ & $153.1 \pm 62.1$ & $150.0 \pm 46.3$ & 0.75 \\
\hline $\mathrm{HbA}_{1 \mathrm{c}}(\%)^{*}$ & $7.8 \pm 2.3$ & $7.8 \pm 2.0$ & 0.95 \\
\hline Systolic blood pressure (mmHg) & $134.5 \pm 15.0$ & $143.2 \pm 25.8$ & 0.04 \\
\hline Diastolic blood pressure $(\mathrm{mmHg})$ & $76.9 \pm 10.2$ & $79.1 \pm 11.5$ & 0.29 \\
\hline \multirow[t]{2}{*}{ Heart rate $(\mathrm{bpm})$} & $76.9 \pm 11.3$ & $79.9 \pm 13.2$ & 0.18 \\
\hline & n (\%) & n (\%) & OR (95\% CI) \\
\hline $\begin{array}{l}\text { Gender } \\
\text { Male } \\
\text { Female }\end{array}$ & $\begin{array}{l}33(61) \\
55(74)\end{array}$ & $\begin{array}{l}21(39) \\
19(26)\end{array}$ & $\begin{array}{c}\text { Reference } \\
0.54(0.25-1.16)\end{array}$ \\
\hline $\begin{array}{c}\text { Pharmacy } \\
\text { A } \\
\text { B } \\
\text { C } \\
\text { D } \\
\text { E } \\
\text { F }\end{array}$ & $\begin{array}{c}19(15) \\
9(7) \\
15(12) \\
9(7) \\
12(9) \\
24(19)\end{array}$ & $\begin{array}{l}4(3) \\
6(5) \\
6(5) \\
9(7) \\
7(5) \\
8(6)\end{array}$ & $\begin{array}{c}\text { Reference } \\
3.17(0.71-14.1) \\
1.90(0.45-7.98) \\
4.75(1.15-19.65) \\
2.77(0.67-11.52) \\
1.58(0.41-6.06)\end{array}$ \\
\hline \begin{tabular}{|l} 
Diabetic Drugs \\
Biguanides \\
Insulins \\
Sulfonylureas \\
DPP-4 inhibitors \\
TZD
\end{tabular} & $\begin{array}{c}57(68) \\
32(58) \\
33(70) \\
10(67) \\
4(67)\end{array}$ & $\begin{array}{l}27(32) \\
23(42) \\
14(30) \\
5(33) \\
2(33)\end{array}$ & $\begin{array}{l}1.13(0.51-2.50) \\
2.37(1.10-5.08) \\
0.90(0.41-1.96) \\
1.11(0.35-3.50) \\
1.11(0.19-6.30)\end{array}$ \\
\hline
\end{tabular}

Citation: Jiménez-Ramírez FJ, Hernandez JJ, Perez R, Lorenzo-Gonzalez W, Perez-Caban Y, et al. (2015) Community Pharmacy Centered Rural Mobile Diabetic Retinopathy Screening Service. J Endocrinol Diab 2(2): 8. DOI: http://dx.doi.org/10.15226/2374- 


\begin{tabular}{|c|c|c|c|}
\hline Hypertension Drugs & & & \\
\hline ACE-I/ARB & $58(63)$ & $34(37)$ & $2.93(1.11-7.76)$ \\
\hline$\beta$-blockers & $13(62)$ & $8(38)$ & $1.44(0.55-3.82)$ \\
\hline Thiazides & $10(62)$ & $6(38)$ & $1.38(0.46-4.09)$ \\
\hline СCB & $9(64)$ & $5(36)$ & $1.25(0.39-4.01)$ \\
\hline Loop diuretics & $3(30)$ & $7(70)$ & $6.01(1.47-24.64)$ \\
\hline Central $\alpha 2$ agonists & $2(50)$ & $2(50)$ & $2.26(0.31-16.67)$ \\
\hline$\alpha 1$ adrenergic blockers & $1(33)$ & $2(67)$ & $4.58(0.40-52.04)$ \\
\hline Hyperlipidemia Drugs & & & \\
\hline Statins & $39(63)$ & $23(37)$ & $1.70(0.80-3.62)$ \\
\hline Fibrates & $4(67)$ & $2(33)$ & $1.11(0.19-6.30)$ \\
\hline Combination & $1(100)$ & $0(0)$ & $\mathrm{NA}^{\dagger}$ \\
\hline Antiplatelet Drugs & & & \\
\hline Aspirin & $33(59)$ & $23(41)$ & $2.54(1.05-4.83)$ \\
\hline Combination & $4(67)$ & $2(33)$ & $1.11(0.19-6.30)$ \\
\hline Clopidogrel & $1(100)$ & $0(0)$ & $\mathrm{NA}^{\dagger}$ \\
\hline
\end{tabular}

${ }^{n} \mathrm{n}=48$ for $\mathrm{HbA}_{1 \mathrm{c}} ;{ }^{\dagger} \mathrm{NA}=$ Not able to calculate a chi-square test because some cells were empty.

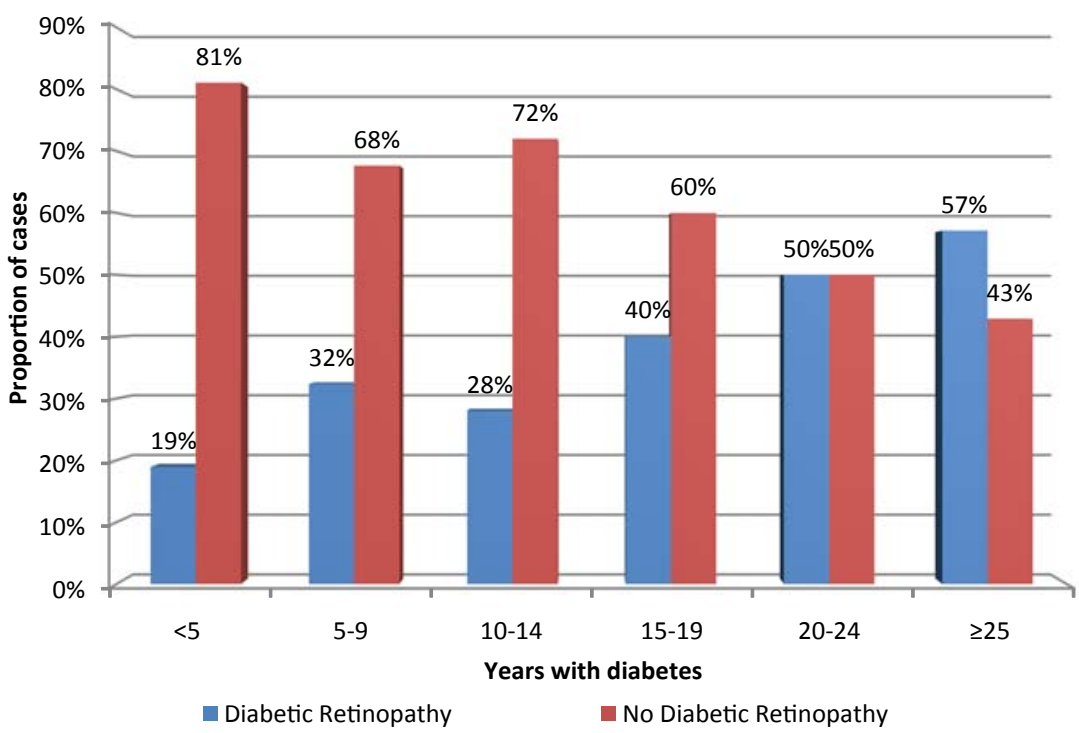

Figure 3: Prevalence of diabetic retinopathy stratified by the number of years since the first diagnosis of diabetes.

160 new suspected eye disorders identified among 101 (79\%) of the 128 participants through the eye screening sessions at the community pharmacies were successfully referred to the participants preferred eye care specialist for appropriate treatment. Future research should evaluate the proportion of participants who indeed attended to their preferred eye care specialist visit, and if in fact the preliminary diagnosis made at the community pharmacy is confirmed and treated.

The distribution of participants' socio-demographic and clinical characteristic was substantially homogenous across all six participant community pharmacies. In terms of a preliminary diagnosis of DR only one of the participant pharmacies (Pharmacy A) was found to have a statistically significant lower prevalence than the other 5 pharmacies (Table 2). It is important to bring up this point since allocation of resources and efforts for future interventions should be based on need.

The overall prevalence of DR in this study was 31\%. The
Table 3: Adjusted Logistic Regression Model to Explore Potential Associations between the Presence of Diabetic Retinopathy and other Covariates among Subjects with Diabetes Mellitus (n=128).

\begin{tabular}{|l|c|c|}
\hline Variables & Odds Ratio & $\mathbf{9 5 \%}$ CI \\
\hline $\begin{array}{l}\text { Age group } \\
\mathbf{2 6 5} \text { years old }\end{array}$ & 0.35 & $0.12-1.07$ \\
\hline $\begin{array}{l}\text { Gender } \\
\text { Female }\end{array}$ & 0.39 & $0.16-0.99$ \\
\hline Body Mass Index (kg/m $\left.\mathbf{m}^{\mathbf{2}}\right)$ & 1.00 & $0.93-1.09$ \\
\hline $\begin{array}{l}\text { Duration of diabetes } \\
\text { (years) }\end{array}$ & 1.08 & $1.02-1.15$ \\
\hline $\begin{array}{l}\text { Systolic Blood Pressure } \\
\text { (mmHg) }\end{array}$ & 1.04 & $1.02-1.07$ \\
\hline Loop diuretics & 6.42 & $1.35-30.60$ \\
\hline ACE-I or ARB & 1.74 & $0.56-5.39$ \\
\hline Aspirin & 1.80 & $0.73-4.43$ \\
\hline
\end{tabular}


prevalence of BDR, the early stage of non-proliferative DR, and proliferative DR, was $30 \%$ and $2 \%$, respectively. Results of an earlier study conducted in Puerto Rico by Jiménez-Ramírez and Pérez [8] reported a lower prevalence of DR (22\%). The Beijing Communities Diabetes Study: Prevalence and Risk Factors for Diabetic Retinopathy [12] also reported a lower prevalence of DR $(24.7 \%)$ than the one reported in this study. Different sampling methods, screening techniques used to evaluate the participants retina, and inclusion and exclusion criteria could explain the differences in DR prevalence between these three studies.

Baseline characteristics and laboratory data were collected to determine which risk factors could be predictive for the development of DR in the Puerto Rican population. Of these variables, male gender, longer duration of DM, use of loop diuretics and higher systolic blood pressure were found to be significantly associated with DR $(P<0.05)$. The tendency showing that DR prevalence increases as the time since first diagnosis of DM increases (OR=1.08; 95\%CI 1.02-1.15) is consistent with the results of the Beijing study, in which DR was present in $40.6 \%$ of people with a duration of DM longer than 15 years [12]. Although age was found to be non-significantly associated with DR (OR=0.35; 95\%CI 0.12-1.07), a trend was found where patient's $\leq 65$ years were at greater risk of presenting DR than older patients.

A limitation of our study is the lack of essential laboratory data such as complete lipid panel, Serum Creatinine (SCr) and Blood Urea Nitrogen (BUN) levels, which were not possible to collect. Lack of complete information on all the medications the participants were taking was another limitation to our study. The current study leads to the development of future research opportunities, such as following up with all patients with a new diagnosis of DR to confirm if they were evaluated and treated by their eye care specialist. Patient's knowledge acquired through the educational and screening sessions at the community pharmacy, and the behavioral change related to performing an annual eye exam should be evaluated. Patient perception of the pharmacist's role in prevention and early identification of DR should also be evaluated in future research.

It has been demonstrated that glucose control contributes significantly to a decrease in DR progression to severe vision loss $[6,13]$, and early diagnosis and treatment of proliferative DR prevent progression to blindness, permanent disability or even death $[3,4]$. Authors expect this study leads to an increase in educational sessions in DR and encourage the pharmacist in the community pharmacy setting to serve as a healthcare provider who address patients' lack of knowledge and provide them screening opportunities. Furthermore, the results of this study suggest that an extensive study in Puerto Rico should be carried out to establish the true prevalence of DR and risk factors for the development of DR among the population with DM.

Most of the economic burden associated with DM is due to the development of vascular complications, including retinopathy. With a proper management and treatment, vision loss can be reduced by $90 \%$ in patients with severe DR [6]. In this study, 79\% of the participants had a suspected diagnosis of an eye disorder. Enhancing awareness about eye health and the importance of an annual screening with an eye specialist is extremely important in this population. Early detection of these conditions will reduce the total burden of DM, including medical costs, which will have an economic impact on the overall productivity of Puerto Rico. Pharmacist's contribution in the early diagnosis and management of DR can have a high impact. They can educate patients with DM on appropriate screening tests to promote an early diagnosis and treatment of DM-related complications. Furthermore, pharmacists can inform patients about ways to improve DM' treatment and outcomes, to promote an early diagnosis of DM and to prevent or delay the onset of DM, thus reducing the burden of DR and other diabetes-related complications.

Findings suggest that there are people with DM with undiagnosed DR at great risk of severe vision loss if prompt diagnosis is not done. The pharmacist, as an accessible healthcare professional, can contribute to enhance awareness of the importance of an annual eye exam and to increase the knowledge about DR and other eye conditions in the general population. In conclusion, this study demonstrates that community pharmacists play an important role in the education, prevention, and early detection of diabetes-related complications. Using a distant or mobile service, in collaboration with an ophthalmologist, is a useful tool to provide access to an annual eye exam to the population with DM in rural areas with poor access to appropriate health care and contribute to the early diagnosis and treatment of DR. The community pharmacy is an excellent setting where pharmacists can inform, educate, and contribute to the prevention of the development of DR or its progression to vision loss in people with DM. Community pharmacies in Puerto Rico should embrace this protocol and develop an island-wide medical information network for the telemedicine screening and early treatment of DR, and other preventable conditions in underserved remote or isolated populations.

\section{Declarations}

The study was approved by the University of Puerto Rico (UPR) Medical Sciences Campus Human Research Subjects Protection Office (HRSPO). A descriptive study was conducted onsite at six community pharmacies in rural cities of Puerto Rico: Farmacia Marie in Corozal, Farmacia La Aurora in Yauco, Farmacia Del Pueblo in Barranquitas, Farmacia San José in Lares, Farmacia Hospital General Castañer in Lares and Farmacia Policlínica Castañer in Adjuntas. Each pharmacy signed an agreement to collaborate with the UPR School of Pharmacy on conducting the study, which consisted of a DR open educational session and a screening evaluation.

Authors disclose no conflict of interest, related to affiliations, honoraria or other personal or professional interest present in this manuscript.

\section{References}

1. Lamoureux EL, Wong TY. Diabetic retinopathy in 2011: further insights from new epidemiological studies and clinical trials. Diabetes 
Care. 2011; 34(4):1066-1067. doi: 10.2337/dc11-0225.

2. National Eye Institute, Facts About Diabetic Retinopathy. [cited 2012 November 15]; Available from: http://www.nei.nih.gov/health/ diabetic/retinopathy.asp.

3. Klein R, Knudtson MD, Lee KE, Gangnon R, Klein BE. The Wisconsin Epidemiologic Study of Diabetic Retinopathy XXIII: the twentyfive-year incidence of macular edema in persons with type 1 diabetes. Ophthalmology. 2009; 116(3):497-503. doi: 10.1016/j. ophtha.2008.10.016.

4. Olayiwola JN, Sobieraj DM, Kulowski K, St Hilaire D, Huang JJ. Improving diabetic retinopathy screening through a statewide telemedicine program at a large federally qualified health center. J Health Care Poor Underserved. 2011; 22(3):804-816. doi: 10.1353/ hpu.2011.0066.

5. Centers for Disease Control and Prevention. Prevalence and Trends Data: Diabetes - 2010. Have you ever been told by a doctor that you have diabetes? 2010. Available from: http://apps.nccd.cdc.gov/ BRFSS/list.asp?cat=DB\&yr=2010\&qkey=1363\&state=All.

6. Centers for Disease Control and Prevevntion, National diabetes fact sheet: national estimates and general information on diabetes and prediabetes in the United States, 2011. Department of Health and Human Services, Editor.

7. Pérez CM, Febo-Vázquez I, Guzmán M, Ortiz AP, Suárez E. Are adults diagnosed with diabetes achieving the American Diabetes Association clinical practice recommendations? P R Health Sci J. 2012; 31(1):1823.

8. Jiménez-Ramírez F, Pérez R. Diabetic retinopathy education and screening at the community pharmacy in Puerto Rico. P R Health Sci J. 2011; 30(3):139-144.

9. Lam CS, Benzie IF, Choi SW, Chan LY, Yeung VT, Woo GC et al. Relationships among diabetic retinopathy, antioxidants, and glycemic control. Optom Vis Sci. 2011; 88(2):251-256. doi: 10.1097/ OPX.0b013e318208494a.

10. Shotliff $\mathrm{K}$, Balasanthiran, Diabetic retinopathy and eye screening. Practice Nurse. 2009. 38(9):26-28.

11. American Diabetes Association. Standards of medical care in diabetes--2013. Diabetes Care. 2013; 36 Suppl 1:S11-66. doi: $10.2337 / \mathrm{dc} 13-\mathrm{S} 011$.

12. Xu J, Wei WB, Yuan MX, Yuan SY, Wan G, Zheng YY, et al., Prevalence and risk factors for diabetic retinopathy: the Beijing Communities Diabetes Study 6. Retina. 2012; 32(2):322-329. doi: 10.1097/ IAE.0b013e31821c4252.

13. Turner R. Intensive blood-glucose control with sulphonylureas or insulin compared with conventional treatment and risk of complications in patients with type 2 diabetes (UKPDS 33). UK Prospective Diabetes Study (UKPDS) Group. Lancet. 1998; 352(9131):837-853 